\title{
A holistic approach for the optimization of offshore wind farm layouts considering cable layouts
}

\author{
Ilayda $\mathrm{Ulku}^{1 *}$, Cigdem Alabas-Uslu ${ }^{2}$ \\ ${ }^{1}$ Istanbul Kultur University, Industrial Engineering Department, Istanbul, Turkey \\ ${ }^{2}$ Marmara University, Industrial Engineering Department, Istanbul, Turkey
}

\begin{abstract}
A wind farm, mainly, is composed of a set of turbines, one or more transmitters and a set of electrical cable connections between turbines and transmitters. Determination of turbine locations within the farm to maximize total power generation is called turbine location (TL) problem. Relative turbine positions affect the amount of overall energy because of wake effects. Determination of cable connections among turbines and transmitters to collect produced energy by turbines at transmitters is called cable layout (CL) problem. While TL problem is directly effective on the total energy production in the farm, CL problem indirectly affects the total energy due to the power losses. In the literature, TL and CL problems are solved sequentially where the layout found by solving of TL is used as an input of CL problem. To minimize wake effects in TL problem, distances between turbine pairs should be increased, however, as the distances are increased the cable cost increases in CL problem. A new mathematical model is developed to deal with simultaneously solving of TL and CL problems. A set of test instances are used to show the performance of the proposed model. The experiments show the practical use of the proposed holistic model.
\end{abstract}

\section{Introduction}

Instead of importing energy at high cost, investment cost of renewable energy which consists of research and development, plant installation, technology, materials and manpower can be afforded. Wind energy becomes a key source of renewable energy because of its high potential to provide the required energy capacity.

Selection and determination of the wind farm site, determination of turbine locations (TL) on the wind farm, designing electrical infrastructure and cable layout (CL) are the main design problems in the installation of an offshore wind farm $[2,3,5,9,10,11]$. TL and CL problems, in the literature, are solved in sequentially due to the difficulties in solving both the problems. Wake effects are minimized by increasing the distances between turbine pairs to generate maximum total energy in TL problem. On the other side, as the distances between turbines increase, the total cable cost also increase. Therefore, objectives of TL and CL problems are conflicting and solving of these problems concurrently may results in more cost-effective wind farms. In a wind farm, the region of reduced and changed wind speed behind the wind turbines is called wake region. If a turbine is in the wake region of one or more turbines, energy production of the effected turbine reduces. One of the most popular wake models used in the related literature is developed by Jensen [1] in 1983. This wake model is based on the distance behind the rotor and assumes that the wake linearly expands.

\section{Literature Review}

TL problem was defined firstly by Mosetti et al. [2] and Jensen's wake decay model was used considering various wind speeds and directions. They developed a genetic algorithm by using a 10x10 grid of possible turbine locations to maximize the ratio of power to installation cost of turbines. Grady et al. [3] obtained better results than Mosetti et al. [2] by using the same example cases and same wake effect. Turner et al. [4] proposed two mathematical models; a quadratic-integer model and a linear-integer programming model for TL problem. They used an interaction matrix prepared in advance to involve wake effects in to the proposed mathematical models. Ulku and Alabas-Uslu [5] developed a new nonlinear mathematical model to solve wind farm layout problem not only for minimization of cost per power under the multiple wake effects but also for maximization of total power production. Their mathematical model has totally unimodularity property which eliminates the binary variables of possible turbine locations. Although their model has nonlinear objective function, the problem becomes to be simpler compared to have both integer variables and nonlinear functions.

CL problem, designing of electrical cable layout between wind turbines and transformer, becomes another major challenge, after designing optimal turbine layouts. Especially for offshore wind farms, $20 \%$ of total installation cost is resulted from cable and cable installation cost. Total cable cost is proportional with the

*Corresponding author: i.karabulut@iku.edu.tr 
length of total cables installed among turbines and transmitter [6-10]. The cable cost can be decreased in overall up to $10 \%$ by optimizing the cable layout problem [11]. As the power flow is unsplittable [8], the power (energy) generated by a turbine is transferred to another turbine or transmitter through only one capacitated cable. Therefore, a solution to CL problem must be at least a minimum spanning tree with capacitated cables. Since the minimum spanning tree problem with capacitated arcs is an NP-hard problem, CL problem is also an NP-hard problem [12]. That's why, numerous studies to solve CL problem have used heuristic methods [13-16]. On the other hand, several studies are based on exact optimization models such as mixed integer linear programming (MILP) models [6, 8, 10, 17-20]. Hertz et al. [8] included parallel cables in their study that can be installed in the same dug hole for onshore wind farms. Fischetti and Pisinger [6] focused on the power losses and non-crossing cables of multiple types and presented both exact solutions using a MIP and heuristic solutions using a hybridization of MIP and heuristic rules. As both TL and CL problems are hard to solve optimally, there is no any study that considers turbine location decisions, cable layout decisions and energy flow decisions through the layout simultaneously [21-23]. All of the existing studies in the literature solve these problems consecutively. Pillai [21] proposed a cable layout optimization problem which is a sub-problem of the turbine layout problem. He included cable connections, the placement of the transformer location to show the effect on the annual energy production and the layout cost. Wedzik and Szypowski [22] developed two-step optimization algorithm to solve first cable layout to minimize the total length of the network, then cable crossing is eliminated through the connected turbines where turbine positions are obtained at the earlier stage. Also, Fischetti [23] focused on the optimal design of wind turbine layout and cable layout. The result of optimal turbine positions was used as an input data for solving of cable layout to minimize total cable cost.

In this study, we present a MINLP model to solve TL problem under multiple wake effect condition and CL problem under multiple cable type condition, simultaneously to provide better solutions to these two conflicting problems. Main contributions of this study are two-fold. First of all, offshore wind farms are rather expensive to invest and any improvement in the investment cost will be quite valuable. Since this study aims to develop optimization-based solution approach, there will be cost savings for a planned wind farm comparing to existing methodologies. Last but not least, as our knowledge, there is no any study in the related literature which considers the two main decision problems (TL and CL) synchronically. Therefore, our study will also contribute to the literature from perspective of operational research.

The rest of this paper is organized into the following. The mathematical model approach is introduced in section 2 at first while section 3 specifies computational results of the proposed approach to show performance of the presented model on different problem instances. Finally, section 4 provides the concluding remarks.

\section{Proposed Mathematical Programming Framework for the Holistic Approach}

The developed model in this study to create a holistic approach for the both TL and CL problems is given through Eq. (1) - Eq. (34). A graph $G=(V, A)$ is used to define the set of turbines under $V$ and a single transmitter in the wind farm while $A$ is the set of possible connections between each pair of nodes in $v \in V$. The wake enlargement, $\alpha$ is calculated in Eq. (1) where the coefficients $z$ symbolizes the hub height and $z_{0}$ represents the surface roughness [1].

$$
\alpha=\frac{0.5}{\ln \frac{Z}{z_{0}}}
$$

The wind speed, $u_{i j}$ is determined with Eq. (2) according to Jensen's wake model as $u_{0}$ is the ambient wind speed. The velocity deficit, $V d_{i j k l}$, in the wind speed due to the turbine located at $(k, l)$ is given in Eq. (3) by using axial induction factor $a$ in Eq. (4) which is dependence of thrust coefficient, $C_{T}$ and rotor radius $r$ [24]. $A_{i j k l}$ is the overlapping area between the swept area of turbine $(i, j)$ and wake area of turbine $(k, l)$. If turbine $(i, j)$ is completely in the wake of turbine $(k, l)$, then $A_{i j k l}=A_{i j}[5]$.

$$
\begin{gathered}
u_{i j}=u_{0}\left(1-\mathrm{V} d_{i j k l}\right) \\
V d_{i j k l}=\frac{2 a}{\left(1+\frac{\alpha D_{i j k l}}{r}\right)^{2}} \frac{A_{i j k l}}{A_{i j}} \\
a=0.5\left(1-\sqrt{1-C_{T}}\right)
\end{gathered}
$$

\subsection{Multiple Wake Effects}

When a turbine is located at position $(i, j)$ and affected by more than one turbine's wake, there occurs a multiple wake effect. The sum of the squares of each single velocity deficits generated by each wake is equal to $V d e f_{i j}$ as given in Eq. (5) [1]. Thus, the residual velocity $u_{i j}$ is calculated in Eq. (6).

$$
\begin{gathered}
\mathrm{Vdef}_{i j}=\sqrt{\sum_{i=1}^{N} \sum_{j=1}^{N} \sum_{k=1}^{N} \sum_{l=1}^{N} \mathrm{~V}_{i j k l}^{2}} \\
u_{i j}=u_{0}\left[1-{\left.\mathrm{V} d e f_{i j}\right]}^{2}\right.
\end{gathered}
$$

Eq. (7) is used to calculate the power obtained by each individual wind turbine $(i, j)$ where $\mathrm{A}$ is cross-sectional area, $\rho$ is air density, and $\eta$ is turbine efficiency. When velocity deficits are included into the power calculation, Eq. (7) is transformed to Eq. (8).

$$
P_{i j}=\eta \frac{1}{2} \rho \mathrm{A} u_{i j}^{3}
$$




$$
\begin{aligned}
P_{i j} & \eta \frac{1}{2} \rho \mathrm{A}\left[u_{0}[1\right. \\
& -\sqrt{\left.\left.\sum_{i=1}^{N} \sum_{j=1}^{N} \sum_{k=1}^{N} \sum_{l=1}^{N} V d_{i j k l}^{2}\right]\right]^{3}} \\
A T C & =(T C O S t) T N\left(\frac{2}{3}+\frac{1}{3} e^{-0.00174(T N)^{2}}\right)
\end{aligned}
$$

The objective function as defined in Eq. (11) includes financial issues that is considered as cost of investment per power unit. The annual cost of investment includes the annual total cost of cables in Eq. (10) and annual total cost of turbines in Eq. (9) that is widely used in the literature and adapted from Mosetti et al [2]. A grid size of size $N x N^{\prime}$ is used to represent the wind farm area where each cell $(I, j)$ represents a possible turbine location. An interaction matrix of size $J=\left(N^{2}\right) x\left(N^{\prime 2}\right)$ is generated a priori by using equations (1-5) where all possible multiple wake effects are included. There is $J-1$ interaction between each possible turbine location $(i, j)$ and other possible turbine locations. A cable of type $r, r \in R$ has cable capacity $\mathrm{Cap}_{r}$, and there exist a Cost $_{r}$ according to the cable capacity between connected turbines at location $(i, j)$ and $(k, l)$. For the given $N x N^{\prime}$ grid size the location of the transmitter is assumed to be known in advance. Since the generated power by a single turbine can leave the turbine with a single cable, the generated power can be transferred as one power unit from that turbine. In addition, cable connections to the transmitter is restricted with a given maximum cable number $C$ due to the technical specifications of the transmitter. Parameters of the problem which are known in advance are listed below: $\mathrm{Cap}_{r}$ : Cable capacity of type $r$ in terms of number of turbines

Cost $_{r}$ : Cost of cable of type $r$ per distance unit

$C$ : Maximum number of cables which can be connected to the transmitter

$M T N$ : The number of turbines

tngh: Maximum allowed distance for each possible turbine position

TCost: Turbine cost

$x_{S S^{\prime}}$ : Transmitter in location $\left(S, S^{\prime}\right)$

$D_{i j k l}$ : Euclidian distance between turbines in locations $(i, j)$ and $(k, l)$ for $i, j, k, l \in \mathrm{I}$

As the aim of the holistic approach is to find costeffective wind farm layouts in terms of both turbine locations and cable connections, the following decision variables of the problem as used.

$T N$ : Total number of turbines

$x_{i j}=\left\{\begin{array}{l}1, \text { there exists a turbine in location }(i, j) \\ 0, \text { otherwise }\end{array}\right.$ $y^{r}{ }_{i j k l}=$

$\{1$, turbines $(i, j)$ and $(k, l)$ are connected with a cable $\{0$, otherwise

flow $_{i j k l}$ : power flow from turbine $(i, j)$ to turbine $(k, l)$

In Eq. (12), spanning tree constraint is introduced to obtain the connection between every pair of nodes turbines and transmitter in the wind farm. The incoming flow to the transmitter is represented with Eq. (13) to guarantee the total number of turbines in the wind farm. The number of turbines used in the wind farm must be at least MTN as shown in Eq. (14). It is provided with Eq. (15) that total number of cables exits from a turbine is either 1 , if a turbine placed in location $(i, j)$, or zero a turbine is not placed in $(i, j)$. Cable connections are avoided if there is another turbine between two turbines in the same row or the same column of the grid design as provided by Eqs. (16-17). Eqs. (18-19) prevent the presence of overlapping cables in each row and column. The cable connection between a pair of turbines is obtained with the maximum allowed distance for each possible turbine position as shown in Eq. (20). Eqs. (2122 ) gives the calculation of outflow from each turbine and similarly inflow to each turbine. Flow conservation is ensured by Eq. (23). The flow amount through a cable is restricted by the capacity of installed cable type as stipulated by Eq. (24). Eqs. (25-26) assure to install at most one cable between a turbine pair. The unidirectionality of the cables is guaranteed with Eqs. (27$28)$. Neither the cable connections nor flow between $(i, j)$ and $(i, j)$ are available as shown in Eqs. (29-30). Also, there is no outflow from the transmitter and it is stipulated in Eq. [31]. With Eq. (32), maximum number of cables which can be connected to the transmitter is represented where the binary restrictions to the variables are represented in Eqs. (33-34).

$$
\begin{aligned}
& \text { ACC } \\
& =\sum_{(i, j),(k, l) \in A} \sum_{r t \in R} \operatorname{cost}_{r} y_{i j k l}^{t} D_{i j k l} \frac{(t)(1+t)^{T}}{T} \\
& \min =\frac{(A C C+A T C)}{P_{i j}} \\
& \sum_{(i, j),(k, l) \in A} y_{i j k l}^{r} \geq T N \quad \forall r \in R \\
& \sum_{k=1}^{N} \sum_{l=1}^{N^{\prime}} \text { flow }_{k, l, S, S^{\prime}}=T N \\
& \sum_{(i, j),(k, l) \in A} x_{i j} \geq M T N \\
& \sum_{k=1}^{N} \sum_{l=1}^{N^{\prime}} \sum_{r=1}^{R} y_{i j k l}^{r}=x_{i j} \quad \forall(i, j) \in \\
& V, i \neq S, J \neq S^{\prime} \\
& y^{r}{ }_{i j i l}=1-x_{i s} \quad \forall(i, j) \text {, } \\
& (i, l) \in V, r \in R, s>j, s<l \\
& y^{r}{ }_{i j k l} \leq 1-x_{s j} \quad \forall(i, j),(k, l) \in V, r \in \\
& R, s>i, s<k \\
& \sum_{l=1, l \neq j}^{N^{\prime}} \sum_{r=1}^{R} y_{i j i l}^{r} \leq x_{i j} \quad \forall(i, j) \in V \\
& \sum_{k \neq i}^{N^{\prime}} \sum_{r=1}^{R} y_{i j k j}^{r} \leq x_{i j} \quad \forall(i, j) \in V
\end{aligned}
$$




$$
\begin{aligned}
& \operatorname{BigM}\left(1-y^{r}{ }_{i j k j}\right) \\
& \geq D_{i j k l} \\
& \text { - tngh } \quad \forall(i, j),(k, l) \\
& \in V, r \in R,(i, j) \\
& \neq\left(S, S^{\prime}\right),(k, l) \neq\left(S, S^{\prime}\right) \\
& \sum_{k=1}^{N} \sum_{l=1}^{N^{\prime}} \text { flow }_{i j k l}=\text { outflow }_{i j} \forall i, j,(i, j) \\
& \sum_{k=1}^{N} \sum_{l=1}^{N^{\prime}} \text { flow }_{k l i j}=\text { inflow }_{i j} \quad \forall(k, l),(i, j) \\
& \neq(k, l) \\
& \text { outflow }_{i j}-\text { inflow }_{i j}=x_{i j} \quad \forall(i, j),(i, j) \neq \\
& \left(S, S^{\prime}\right) \\
& \text { flow }_{i j k l} \leq \sum_{r=1}^{R} \operatorname{Cap}_{r} y^{r}{ }_{i j k l} \quad \forall(i, j),(k, l) \in \\
& V \\
& \sum_{r=1}^{R} y_{i j k l}^{r} \leq x_{i j} \quad \forall(i, j),(k, l) \in V \\
& \sum_{r=1}^{R} y^{r}{ }_{i j k l} \leq x_{j i} \quad \forall(i, j),(k, l) \in V \\
& y^{r}{ }_{i j k l}+y^{r}{ }_{k l i j} \leq x_{i j} \quad \forall(i, j),(k, l) \in V, r \in R \\
& y^{r}{ }_{i j k l}+y^{r}{ }_{k l i j} \leq x_{k l} \quad \forall(i, j),(k, l) \in V, r \in R \\
& y^{r}{ }_{i j i j}=0 \quad \forall(i, j),(k, l) \in V, r \in R \\
& \text { flow }_{i j i j}=0 \quad \forall(i, j) \in V \\
& \text { flow }_{S S^{\prime} i j}=0 \quad \forall(i, j) \in V \\
& \sum_{i=1}^{N} \sum_{j=1}^{N^{\prime}}\left(\sum_{r=1}^{R} y^{r}{ }_{i j s s^{\prime}}\right) \leq C \\
& y^{r}{ }_{i j k l} \in\{0,1\} \quad \forall(i, j),(k, l) \in V, r \in R \\
& x_{i j} \in(0,1) \quad \forall(i, j) \in V
\end{aligned}
$$

\section{Computational Results of Proposed Approach}

To represent the performance of the proposed nonlinear model a set of test problems are generated for a single wind direction from north to south. Fischetti and Pisinger [6] used real wind farms with different cable costs and cable capacities where the cable specifications used in this study are obtained from their study. The proposed MINLP model is tested for three different real wind farms under one wind direction. The wind regime, one wind direction from north to south with constant wind speed of $12 \mathrm{~m} / \mathrm{s}$, is widely used in the wind farm layout literature [2-5, 25]. The cable specifications are given in Table 1. In Table 1, the second column indicates the maximum cable bound to the transmitter. In the third column the number of cable types is shown and it is directly related with the fourth column. Turbine number carried with the cable is represented in fourth column while related cable cost is represented in the last column.

Table 1. Cable Specifications.

\begin{tabular}{ccccc}
\hline Instance \# & $C$ & $r$ & Cap $_{r}$ & Cost $_{r}$ \\
\hline 1 & 10 & 3 & 7 & 370 \\
& & & 11 & 393 \\
& & & 13 & 435 \\
\hline 2 & 4 & 2 & 5 & 407 \\
& & & 10 & 607 \\
\hline 3 & 4 & 2 & 4 & 382 \\
& & & 9 & 630 \\
\hline
\end{tabular}

LINGO software package was used as a solver to solve MINLP model. In table 2, performance of the proposed MINLP model is represented in solving of combined TL and CL problems under study.

Table 2. Performance of Holistic Approach.

\begin{tabular}{ccccccc}
\hline $\begin{array}{c}\text { Instance } \\
\text { Number }\end{array}$ & TN & Power & $\begin{array}{c}\text { Annual Cost } \\
\text { of Cables }\end{array}$ & $\begin{array}{c}\text { Annual Cost } \\
\text { of Turbines }\end{array}$ & $\begin{array}{c}\text { Annual Cost } \\
\text { of Investment }\end{array}$ & $\begin{array}{c}\text { Total Cost of } \\
\text { Investment /Power }\end{array}$ \\
\hline Instance 1 & 30 & 15785.29 & 268959.69 & 27831128.90 & 28100088.58 & 1780.15 \\
\hline Instance 2 & 30 & 15785.29 & 238783.88 & 27831128.90 & 28069912.77 & 1778.23 \\
\hline Instance 3 & 31 & 15870.05 & 432521.90 & 28484972.89 & 28917494.79 & 1822.14 \\
\hline
\end{tabular}

As the cable type is changed the turbine number for the second instance in second column is not changed. The turbine layout as shown in Fig. 1 that is the same for both instance 1 and instance 2 . Thus, annual cost of turbines is also the same for both instance 1 and instance 2. As allowed cable types differ, the optimal cable connections also differ as shown in fourth column of Table 2. The last column represents the total cost of investment per generated power. Instance 1 has least cost per power ratio among the instances represented. The cable capacity and cost according to the capacity of the first instance is preferable in comparison of the presented instances. In Fig. 1, the black line represents cable of type 1, blue line for type 2, and red line for type 3. Although instance 1 and 2 has similar layout, the cable layout differs from each other due to the cable types.

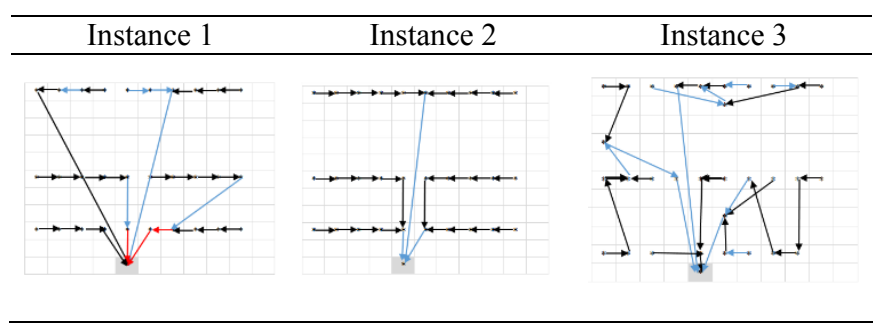

Fig. 1. Cable Connections between Located Wind Turbines. 


\section{Conclusion}

In this study, a holistic approach to solve TL and CL problems is addressed. The combined problem is modelled as a MINLP considering differentiating constraints including economic factors to get close results to the design problems of a real wind farm. Jensen's wake decay model is used for the proposed model to calculate the generated power of each wind turbine. An interaction matrix is used a priori as presented to involve multiple wake effect in the MINLP model. Multiple cable types which differ in capacities and costs are included in the model to provide sending of generated power by the located turbines to a single transmitter assuming that the location of the transmitter is known in advance. Three different case studies were solved and analysed using the MINLP. Although, several constraints to eliminate the crossing cables are imposed in the model to avoid cable damages and costly cable installation, it is observed that the generated results may contain some crossing cables. Therefore, as a future study, we plan to enhance the MINLP to prevent the crossing cables completely.

\section{Acknowledgment}

Participation in this conference was supported under grant [ULEP-2019-U2407/52/03] by Istanbul Kultur University.

\section{References}

1. Jensen, N.O., A note on wind generator interaction. (1983).

2. Mosetti, G., C. Poloni, and B. Daviacco, Optimization of wind turbine positioning in large windfarms by means of a genetic algorithm. J Wind Eng Ind Aerod., 1994. 51: p. 105-116.

3. Grady, S., M. Hussaini, and M.M. Abdullah, Placement of wind turbines using genetic algorithms. Renewable energy, 2005. 30(2): p. 259-270.

4. Turner, S., et al., A new mathematical programming approach to optimize wind farm layouts. Renewable Energy, 2014. 63: p. 674-680.

5. Ulku, I. and C. Alabas-Uslu, A new mathematical programming approach to wind farm layout problem under multiple wake effects. Renewable Energy, 2019. 136C p. 1190-1201.

6. Fischetti, M. and D. Pisinger, Optimizing wind farm cable routing considering power losses. European $\mathrm{J}$ of Operational Research, 2018. 270(3): p. 917-930.

7. Qi, W., Y. Liang, and Z.-J.M. Shen, Joint planning of energy storage and transmission for wind energy generation. Operations Research, 2015. 63(6): p. 1280-1293.

8. Hertz, A., et al., Optimizing the design of a wind farm collection network. INFOR: Information Systems and Operational Research, 2012. 50(2): p. 95-104.

9. Fischetti, M. and D. Pisinger, Optimal wind farm cable routing: Modeling branches and offshore transformer modules. Networks, 2018. 72(1): p. 4259.
10. Cerveira, A., et al., Optimal cable design of wind farms: The infrastructure and losses cost minimization case. IEEE Transactions on Power Systems, 2016. 31 (6): p. 4319-4329.

11. Blanco, M.I., The economics of wind energy. Renewable and sustainable energy reviews, 2009. 13(6-7): p. 1372-1382.

12. Jothi, R. and B. Raghavachari, Approximation Algorithms for the Capacitated Minimum Spanning Tree Problem and Its Variants in Network Design. ACM Trans. Algorithms, 2005. 1: p. 265-282.

13. Gonzalez-Longatt, F., et al., Optimal electric network design for a large offshore wind farm based on a modified genetic algorithm approach. IEEE Systems Journal 2012: p. 164-172.

14. Pillai, A., et al., Offshore wind farm electrical cable layout optimization. Engineering Optimization. 2015. 47(12): p. 1689-1708.

15. Li, D.D., C. He, and Y. Fu. Optimization of internal electric connection system of large offshore wind farm with hybrid genetic and immune algorithm. $3^{\text {rd }}$ International Conference on Electric Utility Deregulation and Restructuring and Power Technologies. 2008. IEEE.

16. Zhao, M., Z. Chen, and F. Blaabjerg, Optimisation of electrical system for offshore wind farms via genetic algorithm. IET Renewable Power Generation, 2009. 3 (2): p. 205-216.

17. Bauer, J. and J. Lysgaard, The offshore wind farm array cable layout problem: a planar open vehicle routing problem. $\mathrm{J}$ of the Operational Research Society, 2015. 66(3): p. 360-368.

18. Berzan, C., et al., Algorithms for cable network design on large-scale wind farms. 2011: Tufts University.

19. Lumbreras, S. and A. Ramos, Optimal design of the electrical layout of an offshore wind farm applying decomposition strategies. IEEE Trans Power Syst, 2013. 28 (2): p. 1434-1441.

20. Banzo, M. and A. Ramos, Optimization of $A C$ Electric Power Systems of Offshore Wind Farms, in Handbook of Wind Power Systems. 2013, Springer. p. 747-771.

21. Pillai, A.C., On the optimization of offshore wind farm layouts. 2017.

22. Wedzik, A., T. Siewierski, and M. Szypowski, A new method for simultaneous optimizing of wind farm's network layout and cable cross-sections by MILP optimization. Applied Energy, 2016. 182: p. 525-538.

23. Fischetti, M., Mixed integer programming models and algorithms for wind farm layout. 2014.

24. Katic, I., J. Højstrup, and N.O. Jensen. A simple model for cluster efficiency. in European wind energy association conference and exhibition. 1987.

25. Marmidis, G., S. Lazarou, and E. Pyrgioti, Optimal placement of wind turbines in a wind park using Monte Carlo simulation. Renewable energy, 2008. 33(7): p. 1455-1460. 